\title{
Morphology Changes of Platinum and Tungsten Carbide by He Plasma Irradiation*)
}

\author{
Yudai TOMITA, Shin KAJITA ${ }^{1)}$, Noriyasu OHNO, Hirohiko TANAKA and Yusuke ICHINO \\ Graduate School of Engineering, Nagoya University, Nagoya 464-8603, Japan \\ ${ }^{1)}$ Institute of Materials and Systems for Sustainability, Nagoya University, Nagoya 464-8603, Japan
}

(Received 28 December 2017 / Accepted 7 May 2018)

\begin{abstract}
Fuzz nanostructure formation on metal by helium plasma irradiation has potential in industrial application due to the increasing in surface area. We have investigated the influence on the surfaces of platinum and tungsten carbide by helium plasma irradiation. On tungsten carbide, fuzz nanostructure was formed when the surface temperature was higher than $1000 \mathrm{~K}$. However, when helium irradiation was conducted at the surface temperature of $940 \mathrm{~K}$ or higher, tungsten carbide changed to $\mathrm{W}$ possibly because of radiation enhanced sublimation. On platinum, fuzz nanostructure was formed when the surface temperature was in the range of $800-1000 \mathrm{~K}$ and the fluence was on the order of $10^{26} \mathrm{~m}^{-2}$. The nanostructure formation mechanism of platinum should be similar to that of tungsten, but incubation fluence of platinum was higher and the growth rate of fuzzy layer was likely to be lower than those of tungsten.
\end{abstract}

(C) 2018 The Japan Society of Plasma Science and Nuclear Fusion Research

Keywords: Helium, fuzz, platinum, tungsten carbide, nanostructure

DOI: $10.1585 /$ pfr.13.3406074

\section{Introduction}

In studies of plasma wall interaction, it is known that fiber-form nanostructures of tungsten (W) are formed by low energy helium (He) plasma irradiation [1]. The nanostructures are called "fuzz". The fuzz structures are formed on various metals such as molybdenum, titanium, iron, and vanadium; the needle-like nanostructures are also formed on silicon by the irradiation [1-4]. In addition, effects on the optical absorption have been reported due to the formation of nanostructures $[5,6]$. These helium plasma irradiation effects have potential for use in industrial applications for example, as catalysts and photocatalysts. Increasing the surface area and optical absorption of catalysts and photocatalysts is advantageous, improving catalytic activities. Improved performance has been reported for water splitting using nanostructure-formed $\mathrm{W}$ [6]. The He plasma exposure is a simple dry process that can make the nanostructure on the surface from tens of minutes to several hours. In order to expand the possibilities for this process to be applied as a catalyst manufacturing method, it is necessary to investigate the influence of the process on materials generally used as catalysts.

Platinum $(\mathrm{Pt})$ is a common material in catalyst industries such as purification of engine exhaust, production of fuel cell electrodes, and modification of photocatalysts [7-9]. However, Pt is scarce and expensive; therefore it is necessary to reduce the consumption of Pt by making nano-sized structures on the surface to enable have

author's e-mail: tomita-yudai@ees.nagoya-u.ac.jp

*) This article is based on the presentation at the 26th International Toki Conference (ITC26). high performance and by developing alternative materials. Tungsten carbide (WC) is one candidate for the alternative materials [10]. WC has been applied as a fuel cell because the valence band of WC is similar to that of Pt $[10,11]$. Unfortunately, neither Pt nor WC has been investigated for effects from He plasma irradiation.

This study focused on the effects of He plasma irradiation on the surface morphology of Pt and WC. For Pt, the influence on the surface morphology was investigated by changing the surface temperature of the samples and He ion fluence. Concerning WC, influence on morphology and molecular structure of the surface was investigated mainly by changing the surface temperature of the samples.

\section{Experimental Setup}

The experiments were performed in the linear plasma device NAGDIS-II (NAGoya DIvertor plasma Simulator). The electron density was on the order of $10^{18} \mathrm{~m}^{-3}$, the electron temperature was $\sim 5 \mathrm{eV}$, and the strength of the magnetic field was $\sim 0.1 \mathrm{~T}$. The surface temperature and incident ion energy were controlled, respectively, by changing the He ion flux to the samples and by biasing voltage of the samples. The surface temperature was measured with a radiation thermometer at a wavelength of $1.6 \mu \mathrm{m}$.

The samples were Pt wires, Pt thin films and WC plates. Pt wires were $5 \mathrm{~mm}$ in length with diameters of $0.5 \mathrm{~mm}$, and spot-welded on tungsten substrates. Pt films were deposited on quartz substrates by RF magnetron sputtering. The thickness of Pt films was $1 \mu \mathrm{m}$ and the size of 
the quartz substrates was $10 \times 10 \mathrm{~mm}$. WC plates size was $11.6 \times 11.6 \mathrm{~mm}$. The radiation thermometer was used to measure the surface temperature at the emissivity of 0.30 for Pt wires, 0.22 for Pt thin films and 0.40 for WC plates. When irradiation was conducted at a surface temperature lower than $1000 \mathrm{~K}$, the samples were situated on a water cooled stage in order to control surface temperature, as heat flux from He plasma is considerably high. The irradiated samples were observed with a scanning electron microscope (SEM). Pt thin films were analyzed by thermal desorption spectrometry (TDS). Molecular structures of WC were analyzed by X-ray diffraction (XRD).

\section{Results and Discussion}

\subsection{He plasma irradiation to tungsten car- bide}

Table 1 summarizes the irradiation conditions of $\mathrm{WC}$, including surface temperature $\left(T_{\mathrm{S}}\right)$ of the samples and incident energy $\left(E_{\mathrm{i}}\right)$. Figure 1 shows the SEM micrograph of WC-3 and WC-7. Fuzzy nanostructures were formed when the surface temperature was higher than $1000 \mathrm{~K}$, the incident ion energy was from 55 to $75 \mathrm{eV}$, and the fluence was on the order of $10^{25} \mathrm{~m}^{-2}$. On the other hand, when the surface temperature was lower than $1000 \mathrm{~K}$, the fuzz structure was not formed, and a rough structure appeared on the surface. The lower limit surface temperature conditions for nanostructure formation were within about 0.3 to 0.4 of the melting point of WC that is $3143 \mathrm{~K}$. Surface temperature dependence was consistent with other materials $[1,2,4]$. The formation conditions and the shape of the fuzz structure were similar to those of $\mathrm{W}$ formed at temperatures higher than about $1000 \mathrm{~K}$ [1].

Figure 2 shows XRD spectra of the irradiated WC samples, namely $\mathrm{WC}-2$, WC-3 and $\mathrm{WC}-4$, and vacuum heated WC without He plasma irradiation. WC-3 and WC-4, which have nanostructures on their surfaces, demonstrated a diffraction peak of $\mathrm{W}$ at about 40 degrees; in particular, WC-4 had weak peaks of WC at about 31 degrees and about 35 degrees. On the other hand, WC-2, which was exposed to He plasma at $550 \mathrm{~K}$ surface temperature, and vacuum heated WC without He plasma irradiation had no diffraction peak of $\mathrm{W}$. The surface structures of both samples were not changed. Carbon (C) peak was not observed in any samples. These results suggest that $\mathrm{C}$ was released from the WC surface by He plasma irradiation, with WC changing to W. Although the sputtering yield of $\mathrm{C}$ is sufficiently higher than that of $\mathrm{W}$ even without nanostructure formation, the phenomenon of $\mathrm{C}$ release would not have resulted only from selective sputtering because no $\mathrm{W}$ peak was observed in the sample irradiated at $550 \mathrm{~K}$.

The $\mathrm{C}$ release may be attributed to radiation enhanced sublimation (RES) [12-14]. RES is a phenomenon unique to C-based material, in which erosion rates increase with an increase in temperature under ion irradiation. The threshold temperature and energy for RES were about
Table 1 The irradiation conditions of WC.

\begin{tabular}{ccccc}
\hline No. & $T_{\mathrm{s}}[\mathrm{K}]$ & $E_{\mathrm{i}}[\mathrm{eV}]$ & Fluence $\left[\mathrm{m}^{-2}\right]$ & $\begin{array}{c}\text { Morphology } \\
\text { changed }\end{array}$ \\
\hline WC-1 & 550 & 55 & $5.7 \times 10^{25}$ & Not changed \\
WC-2 & 820 & 55 & $5.4 \times 10^{25}$ & Not changed \\
WC-3 & 940 & 55 & $5.7 \times 10^{25}$ & Particle \\
WC-4 & 1070 & 57 & $7.4 \times 10^{25}$ & Fuzz \\
WC-5 & 1120 & 55 & $5.7 \times 10^{25}$ & Fuzz \\
WC-6 & 1250 & 55 & $5.7 \times 10^{25}$ & Fuzz \\
WC-7 & 1350 & 75 & $5.7 \times 10^{25}$ & Fuzz \\
\hline
\end{tabular}
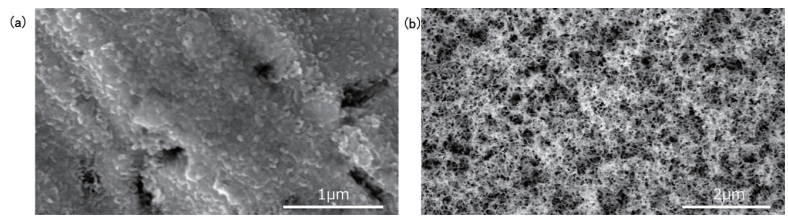

Fig. 1 SEM micrograph on WC irradiated with He plasma (a) WC-3, (b) WC-7.

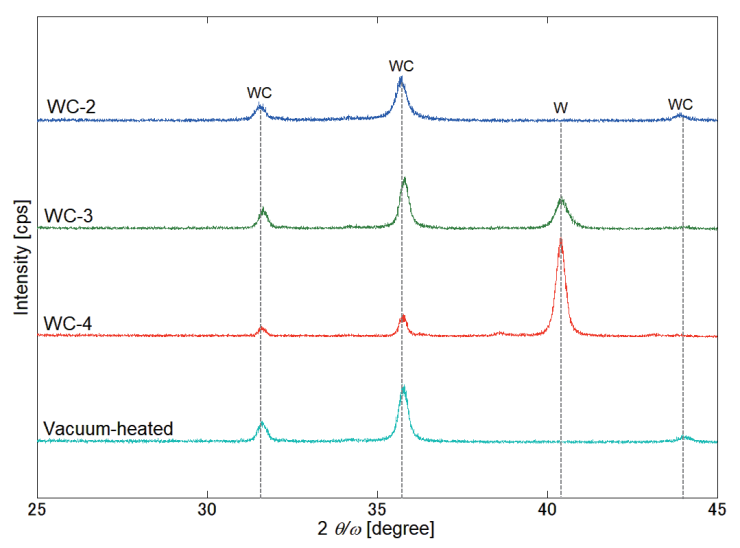

Fig. 2 XRD spectra of the irradiated WC samples and WC heated at $1300 \mathrm{~K}$ for 1 hour in vacuum. The dotted lines are the diffraction peaks of $\mathrm{WC}$ and $\mathrm{W}$, respectively.

$1300 \mathrm{~K}$ and $40 \mathrm{eV}$, respectively $[13,14]$, which was lower than the incident energy in this study. The mechanism of RES was reported by Roth and Möller [14]. It is based on the creation of free interstitial $\mathrm{C}$ atoms by the collision of ions; the interstitial $\mathrm{C}$ atoms diffuse and evaporate from the surface due to temperature rise. In this study, C desorption started at $940 \mathrm{~K}$, which was lower than $1300 \mathrm{~K}$. The formation of He bubbles would have reduced the vacancy density at the surface, and the trap sites might have significantly decreased. These reasons suggest $\mathrm{C}$ emissions in the samples when the surface temperature was lower than $1000 \mathrm{~K}$.

\subsection{He plasma irradiation to platinum}

Table 2 summarizes the irradiation conditions of $\mathrm{Pt}$ wires, the samples were numbered from Pt-1 to Pt-17. 
Table 2 The irradiation conditions of Pt wires.

\begin{tabular}{|c|c|c|c|c|}
\hline No. & $T_{\mathrm{s}}[\mathrm{K}]$ & $E_{\mathrm{i}}[\mathrm{eV}]$ & Fluence $\left[\mathrm{m}^{-2}\right]$ & $\begin{array}{c}\text { Morphology } \\
\text { changed }\end{array}$ \\
\hline Pt-1 & 570 & 75 & $2.9 \times 10^{26}$ & Cone \\
\hline $\mathrm{Pt}-2$ & 630 & 80 & $2.4 \times 10^{24}$ & Cone(partially) \\
\hline Pt-3 & 700 & 75 & $3.3 \times 10^{26}$ & Cone \\
\hline Pt-4 & 810 & 65 & $1.8 \times 10^{26}$ & Fuzz \\
\hline Pt-5 & 840 & 80 & $1.8 \times 10^{25}$ & Fuzz(partially) \\
\hline Pt-6 & 840 & 90 & $1.5 \times 10^{25}$ & Fuzz(partially) \\
\hline Pt-7 & 870 & 90 & $3.9 \times 10^{25}$ & Fuzz(partially) \\
\hline Pt-8 & 910 & 80 & $1.1 \times 10^{26}$ & Fuzz \\
\hline Pt-9 & 930 & 90 & $1.5 \times 10^{25}$ & Fuzz(partially) \\
\hline Pt-10 & 950 & 80 & $2.2 \times 10^{25}$ & Fuzz(partially) \\
\hline Pt-11 & 1000 & 65 & $3.8 \times 10^{26}$ & Fuzz \\
\hline Pt-12 & 1070 & 90 & $2.0 \times 10^{25}$ & Fuzz(partially) \\
\hline Pt-13 & 1080 & 80 & $3.4 \times 10^{25}$ & Fuzz(partially) \\
\hline Pt-14 & 1180 & 55 & $5.0 \times 10^{25}$ & Rough \\
\hline Pt-15 & 1200 & 65 & $2.0 \times 10^{26}$ & Rough \\
\hline Pt-16 & 1320 & 55 & $5.0 \times 10^{25}$ & Rough \\
\hline Pt-17 & 1400 & 55 & $5.0 \times 10^{25}$ & Rough \\
\hline
\end{tabular}

Figure 3 shows the SEM micrograph of Pt-3, Pt-4, Pt11, and Pt-15. Regarding the temperature dependence, the shapes were similar regardless of fluence. Fuzz was formed in the ranges of about 800 to $1000 \mathrm{~K}$. On the other hand, when the surface temperature was lower than $800 \mathrm{~K}$, a cone-like structure was formed. The structure became more round with increasing fluence, suggesting that the cone-like structure was formed by the sputtering. When the surface temperature was higher than $1100 \mathrm{~K}$, the surface structure became bigger and rough. The melting point, $T_{\mathrm{m}}$, of $\mathrm{Pt}$ is $2041 \mathrm{~K}$ and the temperature at which fuzz was formed was within the range of 0.3 to $0.5 T_{\mathrm{m}}$. This dependence was consistent with other materials. The dependence of incident ion energy was not clearly demonstrated.

Figure 4 shows the TDS analysis of a Pt thin film for which the irradiation condition was at a surface temperature of $740 \mathrm{~K}$, incident ion energy was $65 \mathrm{eV}$, and fluence was $1.3 \times 10^{26} \mathrm{~m}^{-2}$. Pressure was evaluated based on the signal intensity of QMS. The sample was heated up to $1000 \mathrm{~K}$ with a heating rate of $0.75 \mathrm{~K} / \mathrm{s}$. The growth mechanism of fuzz structures has been investigated for $\mathrm{W}$ from experimental and theoretical points of view [1, 15]. The fuzz growth is attributed to the diffusion of interstitial $\mathrm{He}$ atoms and the growth of He bubbles. The desorption behavior reflects the motion of interstitial $\mathrm{He}$ atoms and He bubbles. In TDS analysis for W, there are two desorption peaks. One was at around $1000 \mathrm{~K}$, corresponded to the minimum temperature for fuzz growth [16]. Another peak was occur at higher than $2000 \mathrm{~K}$, where no nanostructures were formed on the $\mathrm{W}$ [16]. Moreover molecule dynamics simulation suggested that the He bubble growth and des- (a)

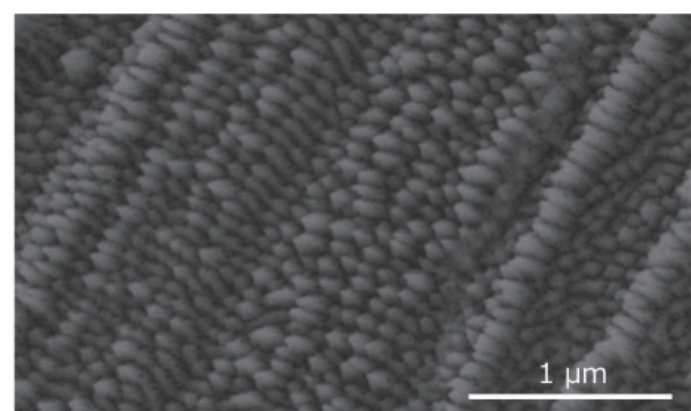

(b)

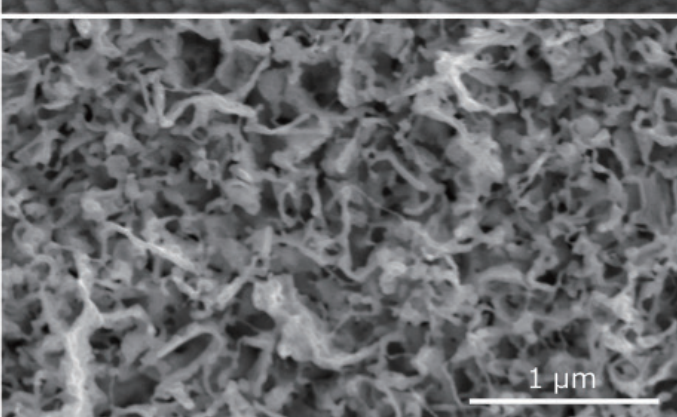

(c)

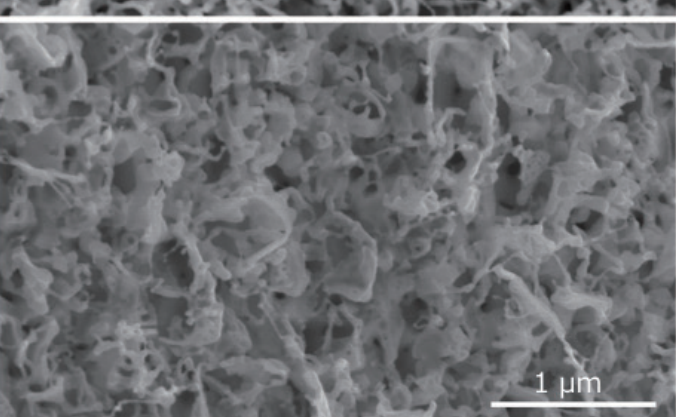

(d)

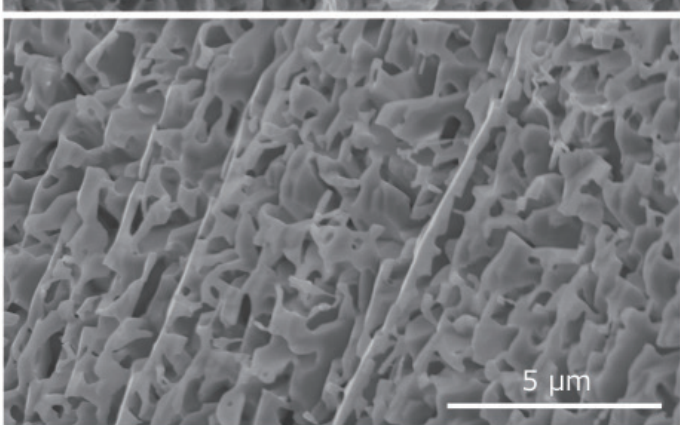

Fig. 3 SEM micrograph on Pt wires irradiated He plasma (a) Pt3, (b) Pt-4, (c) Pt-11, (d) Pt-15.

orption are activated with increasing the temperature [17]. Thus, the He desorption increase from $\sim 800 \mathrm{~K}$ on Pt suggested that He diffusion and growth of He bubbles were activated on Pt surface at the temperature. The desorption temperature corresponded to the minimum temperature at which fuzz structure was formed on $\mathrm{Pt}$ in this study. It seems that the mechanism of fuzz formation and growth of Pt is similar to that of $\mathrm{W}$. The higher peak could not be identified, because we could not increase the temperature of the quartz substrates.

Figure 5 summarizes the relationship between the morphology changes and the irradiation conditions. When the surface temperature was lower than $1100 \mathrm{~K}$ and the flu- 


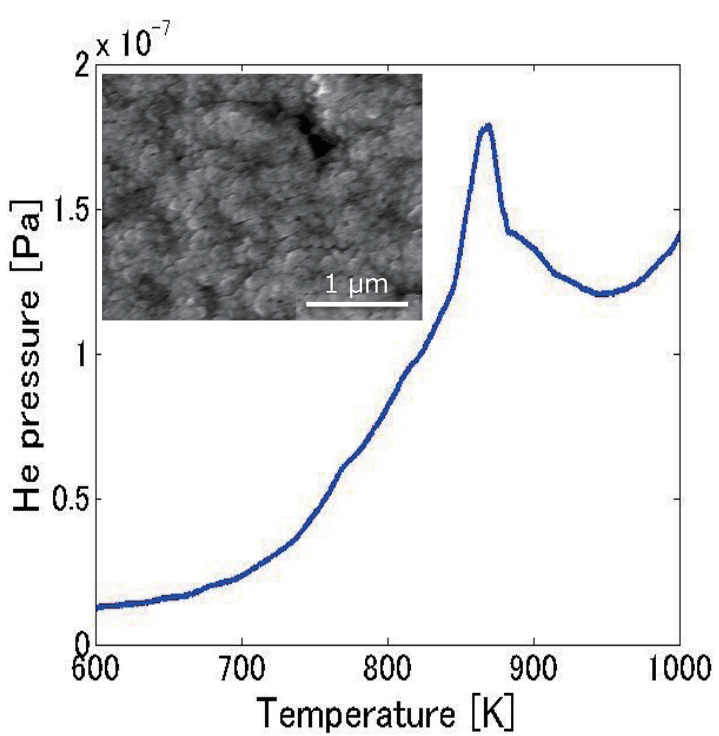

Fig. 4 TDS spectrum from $\mathrm{Pt}$ thin film irradiated with $\mathrm{He}$ plasma at the surface temperature of $740 \mathrm{~K}$. A SEM micrograph is shown in the inset. He pressure was evaluated based on signal intensity of QMS.

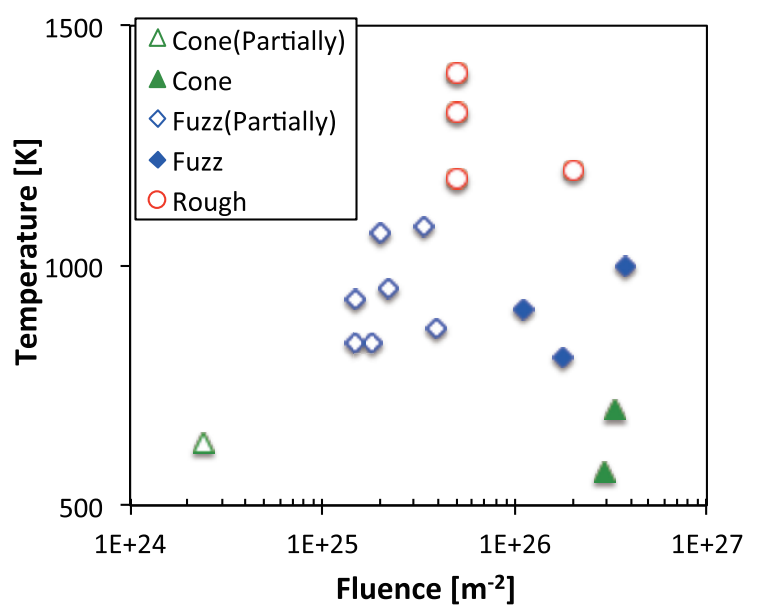

Fig. 5 The surface temperature was plotted against the fluence. The closed markers represent the cases that nanostructures were formed on the whole surfaces.

ence was on the order of $10^{26} \mathrm{~m}^{-2}$, nanostructures were formed on the entire surface. When the fluence was lower than $10^{26} \mathrm{~m}^{-2}$, nanostructures were locally formed, and the surfaces have rough structures and many pinholes were made. Petty et al. reported that the thickness of fuzz layer depended on square root of fluence, and the fluence had threshold, which was called incubation fluence, in order to form fuzz structure [18]. On W, nanostructures were formed on the entire surface at the fluence of $\sim 10^{25} \mathrm{~m}^{-2}$, which was about one order of magnitude lower than that of Pt [1, 18]; also, the incubation fluence of Pt was likely to be about one order of magnitude higher than that of W.

\section{Conclusion}

Surface morphology changes of WC and Pt from the irradiation of He plasma were experimentally investigated. A fuzz structure was formed on the WC surface when the surface temperature was higher than $1000 \mathrm{~K}$. In XRD analysis, the samples, on which the nanostructures were formed, showed weak WC peaks but a strong W peak. This was likely due to radiation enhanced sublimation (RES), and RES was likely promoted at lower temperature because the trap sites of interstitial carbon atoms were decreased by the growth of He bubbles in WC.

Fuzz structures were fully formed on the surfaces of $\mathrm{Pt}$ when the surface temperature was 800 to $1000 \mathrm{~K}$ and the fluence was on the order of $10^{26} \mathrm{~m}^{-2}$. In order to form nanostructures on $\mathrm{Pt}, 10$ to 100 times the He fluence was required compared with the fluence necessary for forming nanostructures on $\mathrm{W}$. The Pt fuzz method can be utilized to nanosizing technology in catalyst industry in future. We plan to investigate the catalic reactivity in future.

\section{Acknowledgment}

This work was supported in part by a Grant-in-Aid for Scientific Research (B) 15 H04229 and a Grant-in-Aid for Exploratory Research 16K13917 from the Japan Society for the Promotion of Science (JSPS).

[1] S. Kajita et al., Nucl. Fusion 49, 095005 (2009).

[2] S. Kajita et al., Appl. Surf. Sci. 303, 438 (2014).

[3] S. Kajita et al., Scientific Reports 6, 30380 (2016).

[4] J.K. Tripathi et al., Appl. Surf. Sci. 378, 63 (2016).

[5] S. Kajita et al., J. Appl. Phys. 113, 134301 (2013).

[6] M. de Respinis et al., ACS Appl. Mater. Interface 5, 7621 (2013).

[7] H. Igarashi et al., J. Electroanal. Chem. 391, 119 (1995).

[8] F. Kapteijn et al., Catal. Today 16, 273 (1993).

[9] C. Wang et al., ACS Appl. Mater. Interface 2, 3373 (2010).

[10] R.B. Lavy and M. Boudart, Science 181, 547 (1973).

[11] R.J. Colton et al., Chem. Phys. Lett. 34, 2, 337 (1975).

[12] V. Philipps et al., J. Nucl. Mater. 179-181, 25 (1989).

[13] R.E. Nygren et al., J. Nucl. Mater. 176\&177, 445 (1990).

[14] J. Roth and W. Möller, Nucl. Instrum. Methods Phys. Res. B 7/8, 788 (1985).

[15] S. Kajita et al., J. Nucl. Mater. 418, 152 (2011).

[16] M. Yajima et al., J. Nucl. Mater. 449, 9 (2014).

[17] A. Lasa et al., Nucl. Instrum. Methods Phys. Res. B, 303, 156 (2013).

[18] T.J. Petty et al., Nucl. Fusion 55, 093033 (2015). 